\title{
Évelyne DIEBOLT et Nicole FOUCHÉ, Devenir infirmière en France. Une histoire atlantique? (1854-1938)
}

Paris, Publibook, 2011, 337 p.

\section{Pierre-Yves Saunier}

\section{OpenEdition}

\section{Journals}

Édition électronique

URL : http://journals.openedition.org/clio/10892

DOI : $10.4000 /$ clio.10892

ISSN : 1777-5299

Éditeur

Belin

Édition imprimée

Date de publication : 31 décembre 2012

ISSN : 1252-7017

Référence électronique

Pierre-Yves Saunier, «Évelyne diebolt et Nicole fouché, Devenir infirmière en France. Une histoire atlantique? (1854-1938) », Clio. Femmes, Genre, Histoire [En ligne], 36 | 2012, mis en ligne le 19 avril 2013, consulté le 22 septembre 2020. URL : http://journals.openedition.org/clio/10892 ; DOl : https:// doi.org/10.4000/clio.10892

Ce document a été généré automatiquement le 22 septembre 2020

Tous droits réservés 


\title{
Évelyne DIEBOLT et Nicole FOUCHÉ, Devenir infirmière en France. Une histoire atlantique? (1854-1938)
}

\author{
Paris, Publibook, 2011, 337 p.
}

Pierre-Yves Saunier

\section{RÉFÉRENCE}

Évelyne DIEBolt et Nicole FouchÉ, Devenir infirmière en France. Une histoire atlantique ?

(1854-1938), Paris, Publibook, 2011, 337 p.

1 Qu'est-ce que les auteures entendent en proposant de tester la validité d'une « histoire atlantique » de la profession d'infirmière en France? Elles veulent montrer que ce qui se passe en matière de nursing en Angleterre ou aux États-Unis a affecté la trajectoire historique des différentes protagonistes des soins infirmiers en France, que l'on considère ceux-ci comme une profession (organisation d'un groupe défini par son activité) ou comme une discipline (enseignée dans des institutions dédiées). Notons au passage qu'elles ne traitent pas du métier (ensemble de gestes accomplis au quotidien). Et elles démontrent en effet, par l'étude des usages rhétoriques, par la reconstitution de la circulation de l'information (revues, correspondance, visites), et par une attention précise aux sites où s'installent des formes d'enseignement proches des écoles-pilotes de Londres ou de la côte Est des États-Unis, que les protagonistes français du développement de l'enseignement infirmier entre 1878 et 1938 pensent avec et contre les expériences anglaises et états-uniennes. Et que les protagonistes de ces expériences s'intéressent aux écoles françaises comme terrain de généralisation de leurs particularités. Ces liens et ces flux donnent lieu à divers types d'usages : observation, adaptation, imitation, différenciation, soumission, mais aussi simple ostentation de la référence étrangère à des fins de légitimation (l'inévitable invocation à Florence Nightingale en étant la forme cardinale). 
2 L'ouvrage identifie clairement un certain nombre de sites et d'actrices qui prennent part à la mise en place de ces liens de référence et d'usage en matière de définition de la figure sociale de l'infirmière, de fonctionnement des écoles de formation des infirmières ou de réglementation de la profession. Avec les auteures, on écoute le Docteur Bourneville tordre les formules mises en place à Saint Thomas' Hospital et dans d'autres écoles londoniennes pour en installer sa version dans le système de l'Assistance Publique parisienne. On suit le travail de Anna Hamilton et de certaines institutions états-uniennes (American Red Cross, associations professionnelles des nurses états-uniennes, Rockefeller Foundation) pour tenter de synchroniser le champ français avec les mouvements en cours dans l'espace états-unien. On assiste aussi au travail d'autres protagonistes françaises, Léonie Chaptal ou Marie Liron d'Airoles, pour découpler cette interdépendance et conserver un certain statu quo, en s'appuyant néanmoins sur des ressources qui elles aussi jouent à travers les frontières (congrès, associations).

3 En faisant ce suivi, Évelyne Diebolt et Nicole Fouché montrent combien un certain nombre de phénomènes 'français' aussi divers que des agences étatiques (le bureau central des infirmières du ministère de la Santé entre les deux guerres), des écoles (la Maison de Santé Protestante de Bordeaux, les écoles municipales de la Ville de Paris fondées à partir de 1878), des structures sanitaires (les dispensaires installés par l'American Red Cross ou la Fondation Rockefeller pendant la Première Guerre Mondiale) inscrivent la présence et la contribution du nursing états-unien ou britannique dans le paysage français. De la même manière, si le décret sur le brevet de capacité professionnelle du 27 juin 1922 est un des produits de l'entregent social et politique de la Française Léonie Chaptal, il doit aussi certains de ses accents, ou des formules qu'il installe, aux propositions faites par les infirmières américaines ayant travaillé en France depuis la Première Guerre mondiale. La limite entre le national et l'étranger, le forain et le domestique, n'est pas si évidente que cela.

4 La démonstration est convaincante car les auteures ne se satisfont pas d'analogies pour traquer l'effet des formes anglaises et états-uniennes sur le développement des écoles ou la conception du métier. Elles suivent des écoles précises, à Paris et en Province ; elles mettent dans le circuit des figures connues (Léonie Chaptal, le Dr Bourneville, Anne Morgan, Marie Adelaide Nutting, Lavinia Dock) aussi bien que des profils moins familiers (Jeanne de Joannis, Juliette Delagrange, Frances Elisabeth Crowell); elles établissent les différentes situations dans lesquelles les propositions qui se mettent en œuvre aux États-Unis et en Angleterre font sentir leur impact; elles expliquent combien ce qui est proposé aux infirmières françaises comme modèle est encore loin d'être la norme aux États-Unis même. Et finalement, si elles ne font pas le récit d'un succès, elles évaluent finement les dimensions de l'impact des entreprises menées par des Françaises ou des organisations états-uniennes pour transposer certaines des formes du projet de professionnalisation mené par les nurses états-uniennes à partir des années 1880-1890. Certes, la tentative de faire du métier d'infirmière une carrière pour des femmes hautement spécialisées, recrutées après des études secondaires, dotées d'un diplôme universitaire et placées dans de hautes positions de responsabilité dans les hôpitaux et les écoles d'infirmières, échoue. Les auteures attribuent cet échec à la coalition tacite entre la République, peu soucieuse d'inscrire la formation de ces jeunes femmes au budget national, et de réformatrices tièdes comme Léonie Chaptal, qui n'adhère pas au projet d'émancipation féminine proposé par les nurses états- 
uniennes. Néanmoins, les diverses expériences menées ont laissé des traces durables (des écoles, des institutions), et ont surtout contribué à ouvrir le cadre des possibles à divers moments de la trajectoire de la profession et de l'éducation infirmière. Ce n'est pas seulement dans le succès que réside l'intérêt des études des phénomènes qui se développent entre et à travers les états nationaux.

Dans leur introduction et leur conclusion, les auteures présentent l'ouvrage comme une synthèse. C'est en effet le cas, puisqu'il reprend et augmente un certain nombre des travaux qu'elles ont effectuées individuellement et collectivement depuis une trentaine d'années. Il met aussi à disposition une considérable bibliographie, ainsi que de nombreuses notices biographiques. Pourtant, l'ouvrage semble plutôt un jalon, et pas seulement parce que son aspect synthétique se perd parfois dans les redites, les détails, les retours en arrière ou une carence de matériaux et travaux à synthétiser qui pousse les auteurs à formuler des hypothèses fragiles. Pour toutes ces raisons, les personnes déjà familières de l'histoire des infirmières y apprendront plus que les autres, car il faut des repères pour lire ce jalon.

6 Jalon aussi parce que ce livre, autour de l'histoire des soins infirmiers, est un rebond distant de cette période des années 1980 où, sous la houlette de Marie-France Collière, Yvonne Knibiehler, Yves Lequin et d'autres historiennes et chercheuses, l'histoire des soins infirmiers avait fait l'objet d'un premier effort conjoint de recherche et de publication. Quarante ans après, il n'est pas anodin de constater que la publication de ce livre est contemporaine de celle de l'ouvrage de Christian Chevandier, Les infirmières parisiennes 1900-1950, dont l'auteur a été un doctorant d'Yves Lequin à Lyon. Nous trouverions-nous en face d'une nouvelle fenêtre où l'étude des infirmières est d'actualité pour les historiennes et historiens français?

7 Jalon encore parce qu'il ouvre, par ses acquis et ses absences, un certain nombre de pistes à développer. Qu'en est-il de la trajectoire professionnelle des infirmières formées dans les "lieux-pilotes" où s'incarne le projet "émancipateur" porté par les consœurs états-uniennes et leurs correspondantes françaises? Où ont-elles porté leur expérience, leur tour de main, leurs méthodes, leur conception de la profession ou de l'éducation infirmière? Que ramènent exactement d'Angleterre ou des États-Unis les jeunes femmes françaises qui vont s'y former ou s'y perfectionner au métier d'infirmière ? En quoi consiste le lien régulier et durable de conseil et d'échanges entre le Bureau Central des Infirmières, les écoles et les 'conseillères techniques' rockefelleriennes? Que se passe-t-il dans des lieux où ont été prodigués, de manière peut-être moins visible que les écoles parisiennes ou la Maison de Santé Protestante de Bordeaux, les avis et l'assistance des collègues états-uniennes: au dispensaire polyvalent de Montpellier, à celui de Paray le Monial, dans le service de santé départemental de Meurthe-et-Moselle, dans les écoles de Strasbourg, de Marseille, ou encore dans les écoles dirigées par des religieuses à Nancy ou à Nantes? Par ailleurs, le fonctionnement des hôpitaux et des services hospitaliers, et les contraintes qu'il fait peser sur la possibilité de réaliser le projet émancipateur du nursing, ne sont pas présents dans Devenir infirmière. Il est vrai que peu d'écoles-pilotes semblent avoir réussi à développer des relations durables avec des services hospitaliers qui donneraient toute leur dimension aux études pratiques des élèves infirmières. Mais plus largement, la manière dont le personnel soignant non diplômé considère le projet émancipateur, très élitiste, est aussi laissée de côté dans Devenir infirmière. Le lire aux 
côtés des travaux de Christian Chevandier, mentionné ci-dessus, s'avèrera donc très complémentaire.

Jalon enfin parce que la perspective triangulaire qui est celle du livre, centré sur les liens, les flux et les relations entre la France, l'Angleterre et les États-Unis, ne doit pas faire oublier que cette histoire de mobilité des normes professionnelles, des savoirs enseignés et des gestes pratiqués ne se borne pas au triangle ci-dessus : les grands et les petits ordres religieux soignants catholiques ont essaimé leurs sœurs et leurs hôpitaux de par le monde depuis le début $\mathrm{du} \mathrm{XIX}^{\mathrm{e}}$ siècle, les missionnaires nightingaliennes ont rayonné sur le domaine anglophone et au-delà, sans oublier d'autres initiatives contemporaines et connexes plus directement liées au Réveil protestant, les diaconesses germaniques et les garde-malades de Madame de Gasparin et de son école de Lausanne. Plus tard, les programmes de la Fondation Rockefeller, de l'American Red Cross, de la League of Red Cross Societies, aussi bien que les contacts des sociétés professionnelles d'infirmières britanniques ou états-uniennes, incluent des relations avec la France aussi bien que dans le reste de l'Europe et bien au-delà. Après la Deuxième Guerre mondiale, l'Organisation Mondiale de la Santé ou le Colombo Plan rejoignent ces entreprises de définition et de diffusion d'un 'universel' du nursing. Ce qui implique que les initiatives soutenues ou initiées en territoire français par ces différents protagonistes prennent sens dans un ensemble d'initiatives, dans des circuits à multiple détente, et pas seulement dans des relations bi- ou trilatérales. Celles-ci, d'ailleurs, ne se déroulent pas vraiment entre des pays mais concernent des individus, des segments professionnels et sociaux, des écoles ou des institutions bien précises. Devenir infirmière, une histoire planétaire ? C'est en tout cas l'espace de la définition de ce devenir qui reste à préciser.

\section{AUTEURS}

\section{PIERRE-YVES SAUNIER}

CNRS, IRICE-UMR 8138 